\title{
Using Mobile Technology to Provide Personalized Reminiscence for People Living With Dementia and Their Carers: Appraisal of Outcomes From a Quasi-Experimental Study
}

Elizabeth A Laird ${ }^{1}$, BSc (Hons), MSc, PhD; Assumpta Ryan ${ }^{1}$, BSc (Hons), MEd, PhD; Claire McCauley ${ }^{1}$, BPharm (Hons), MSc, PhD; Raymond B Bond ${ }^{2}$, BCompSc (Hons), PhD; Maurice D Mulvenna ${ }^{2}$, BSc (Hons), MPhil, PhD; Kevin J Curran ${ }^{3}$, BSc (Hons), PhD; Brendan Bunting ${ }^{4}$, BSc (Hons), MPsychol, PhD; Finola Ferry ${ }^{4}$, BSc (Hons) Psychol, PhD; Aideen Gibson², BCompSc (Hons), MSc

\footnotetext{
${ }^{1}$ Institute of Nursing and Health Research, Ulster University, Derry, United Kingdom

${ }^{2}$ School of Computing, Ulster University, Belfast, United Kingdom

${ }^{3}$ School of Computing, Engineering \& Intelligent Systems, Ulster University, Derry, United Kingdom

${ }^{4}$ Psychology Research Institute, Ulster University, Derry, United Kingdom
}

\section{Corresponding Author:}

Elizabeth A Laird, BSc (Hons), MSc, PhD

Institute of Nursing and Health Research

Ulster University

Northland Road

Derry, BT48 7JL

United Kingdom

Phone: 442871675006 ext 75006

Fax: 442871675493

Email: ea.laird@ulster.ac.uk

\section{Abstract}

Background: Dementia is an international research priority. Reminiscence is an intervention that prompts memories and has been widely used as a therapeutic approach for people living with dementia. We developed a novel iPad app to support home-based personalized reminiscence. It is crucial that technology-enabled reminiscence interventions are appraised.

Objective: We sought to measure the effect of technology-enabled reminiscence on mutuality (defined as the level of "closeness" between an adult living with dementia and their carer), quality of carer and patient relationship, and subjective well-being.

Methods: A 19-week personalized reminiscence intervention facilitated by a program of training and a bespoke iPad app was delivered to people living with dementia and their family carers at their own homes. Participants (N=60) were recruited in dyads from a cognitive rehabilitation team affiliated with a large UK health care organization. Each dyad comprised a person living with early to moderate dementia and his or her family carer. Outcome measurement data were collected at baseline, midpoint, and intervention closure.

Results: Participants living with dementia attained statistically significant increases in mutuality, quality of carer and patient relationship, and subjective well-being $(P<.001$ for all 3$)$ from baseline to endpoint. Carers attained nonsignificant increases in mutuality and quality of carer and patient relationship and a nonsignificant decrease in subjective well-being.

Conclusions: Our results indicate that individual-specific reminiscence supported by an iPad app may be efficient in the context of early to moderate dementia. A robust randomized controlled trial of technology-enabled personalized reminiscence is warranted.

(JMIR Ment Health 2018;5(3):e57) doi: 10.2196/mental.9684

\section{KEYWORDS}

dementia; evaluation; mobile apps; reminiscence; research; technology; mobile phone 


\section{Introduction}

\section{Background}

Dementia is an umbrella term that encompasses at least 40 conditions that feature progressive cognitive decline and are more prevalent in older age. In tandem with international aging demographics, the prevalence of dementia and associated costs have risen substantially. The estimated annual UK cost of dementia is over $£ 26$ billion [1], and this is higher than the combined costs for cancer, stroke, and heart disease. There is increasing evidence that nonpharmacological interventions for the symptoms of dementia can have commensurate effectiveness to pharmacological treatment and may be preferable where medication can cause negative side effects [2-4]. The progressive nature of dementia presents a challenge for families providing care to a relative with this condition [5-7]. Not surprisingly, the World Health Organization (WHO) has prioritized dementia as a global public health concern and has recommended that more research should be undertaken to inform supportive interventions for people living with dementia and their families [8].

Reminiscence refers to a range of psychosocial interventions that prompt memories and has been widely used as a therapeutic approach for people living with dementia and their carers $[9,10]$. Technology-based reminiscence increases opportunities to participate in conversations and enhances the social interactions of people living with dementia and their carers [10]; furthermore, it enables remote reminiscence to be delivered at home [11]. Traditional reminiscence utilizes collections of resources such as memory boxes that can stimulate a range of senses, including touch, taste, and smell. In contrast, technology-based reminiscence is reliant on visual and auditory memory prompts. These limitations may be offset by the portability, mobility, and utility of technology-based reminiscence systems to deliver personalized reminiscence experiences.

A systematic review [12] of technology-supported reminiscence therapy identified 44 papers that met the selection criteria. Although limited by the small sample sizes of some of the studies, the authors concluded that there were benefits to using information and communication technology (ICT) for reminiscence interventions. These benefits include access to rich and engaging multimedia reminiscence materials [13,14], opportunities for people living with dementia to participate in social interactions and take ownership of conversations $[15,16]$, and a reduction in motor deficit-related barriers when interacting with media [16,17]. In the abovementioned review [12], 10 reviewed papers reported on the use of "reminiscence kits" that featured a technological component. Audio was a major component of these reminiscence kits, but impact evaluation was not reported. One study [17] examined the attitudes of older people $(\mathrm{N}=19)$ toward using an iPad to aid reminiscence. Participants in the study were randomly allocated to reminisce using either an iPad or more traditional images and cards. The results from that study indicated that participants enjoyed using the iPad. In a follow-up mixed methods design, a mobile app called "Memory Matters" was developed to promote reminiscence [15]; 18 people living with dementia and 8 family carers were asked to use MM for a period of 4 weeks. Consistent with the findings of a more recent study that explored a similar device [18], the technology-supported reminiscence was favorably evaluated. Family carers enjoyed discussing the early years with their relative, and on several occasions, the people living with dementia shared memories in a direct response to prompts provided by MM. People living with dementia who had only interacted minimally, or who had never spoken before, were observed to interact and support each other while using the app. These findings support the social engagement potential of mobile devices in the context of family caregiving in dementia $[19,20]$.

As dementia progresses, it is common for carers to report a "disappearance of the relationship" $[21,22]$. There is a need to support caregiving relationships in order to protect mental and physical well-being of the carers $[23,24]$. ICT has an important role to play in this endeavor by supporting social connectivity [25]. It is important, therefore, that in studies of technology-based reminiscence, family carers are included in addition to people living with dementia.

Our research team was motivated by the rising acceptability of health apps to develop and test the feasibility of a novel app to deliver personalized reminiscence among people living with dementia and their carers. Consistent with recommendations [12], validated and standard outcome measures were selected for the appraisal of efficacy. Mutuality is a scale that measures closeness in a relationship [26]; WHO-Five Well-Being Index (WHO-5) is a short scale for measuring emotional well-being [27], and Quality of Carer-Patient Relationship scale (QCPR) is a scale of family caregiving [28]. All 3 scales have been tested in dementia research, but not in reminiscence research. This paper contributes to the evidence base by reporting the preliminary efficacy of technology-based personalized reminiscence facilitated by a program of training and an iPad app on mutuality, quality of caregiving relationships, and subjective well-being among people living with dementia and their family carers.

\section{Development of a Reminiscence App}

The size, capacity, and low cost of ubiquitous mobile devices have made them an attractive option for technology-based reminiscence systems. As part of this study, a cross-platform device agnostic tablet app (called InspireD: an acronym for Individual-Specific Reminiscence for People living with Dementia) was developed to facilitate reminiscing activity. The two primary aims of the app were to enable people living with dementia and their family carers to select and store personalized memorabilia (photographs, videos, sounds, music, etc) and to provide easy access to these visual and audiovisual cues to support bespoke reminiscence.

The InspireD app was developed [29] with input from the Reminiscence Network Northern Ireland and a user development group that comprised a total of 7 dyads, with each dyad comprising a person living with dementia and his or her primary caregiver $(n=14)$. The Agile software development approach [30] was adopted to allow the functional prototype to be created early in the development lifecycle, with testing for usability and refinement taking place throughout the development process 
[29]. The app was implemented using Appcelerator Studio (Appcelerator Titanium SDK, US), an Eclipse-based integrated development environment that provides an environment to build, test, package, and publish apps for various platforms, including iOS and Android. The code is written in JavaScript, with native user interface (UI) elements being invoked at runtime. It incorporates local facilities for persistent data storage in SQLite database and facilitates the use of third-party app programming interfaces for Flickr and YouTube (Figure 1).

The app consists of a UI that is usable and responsive across a variety of mobile devices (tablets, mobile phones). It is also possible to use the system on a personal computer or laptop via the Web browser. The main user (and co-users, ie, carers) can upload images, videos clips, and audio clips to the app. SQLite database functionality is used to store and manage data natively.

Figure 1. InspireD app system architecture SQLiteDB SQLite database.
The main UI consists of a simple screen for people living with dementia to upload files with help from a reminiscence trainer or a family caregiver. A multiscreen layout allows users to choose which memorabilia they wish to access: view photos, watch videos, listen to audio files, and browse selected resources (Figure 2).

The design is minimalist, using verbal descriptors as well as images and icons to reinforce and indicate functionality to the user. Data are organized and presented primarily in the form of on-screen menus. The welcome screen is a simple log-in screen where the users confirm their identity by clicking a photo of themselves. The user data are contained within a local SQLite database, which can be easily queried with the reporting services enabled. Multimedia reminiscing resources (photos, videos, and audios) are also stored locally in the app data directory.

InspireD System Architecture

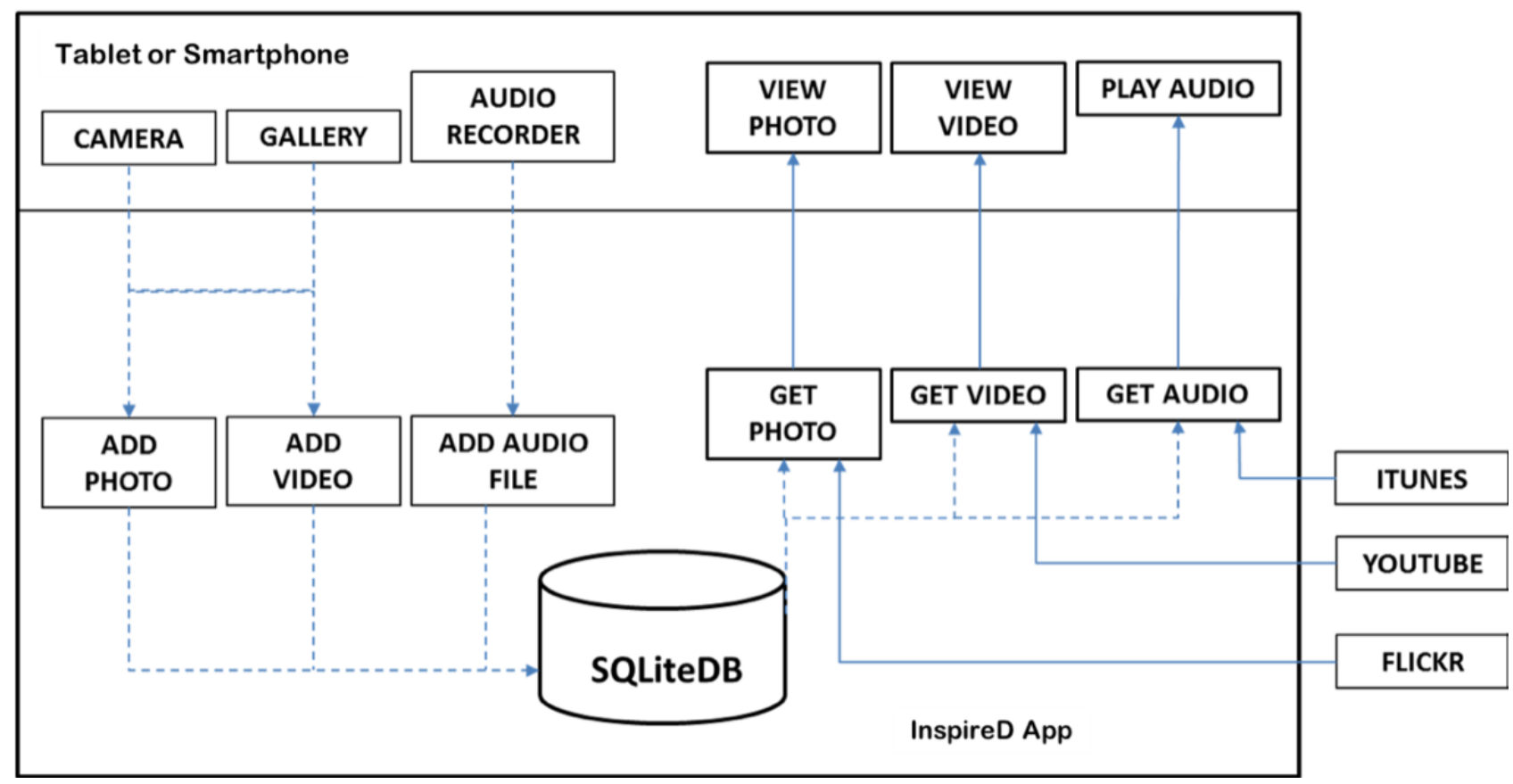

Figure 2. The InspireD reminiscence app user interface.
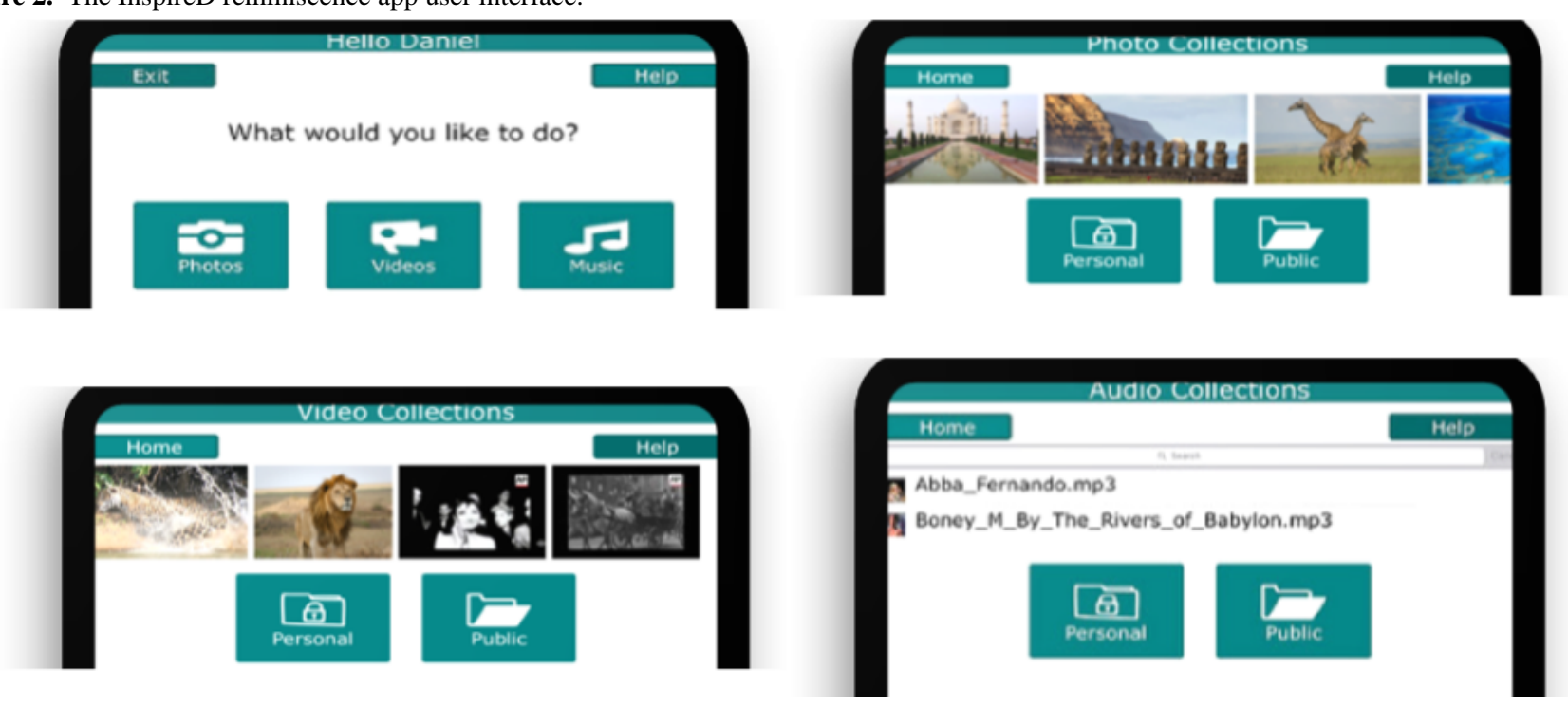
The InspireD app incorporates a logging facility for 5 canonical events. These are entry (logging in), admin (adding a photo, deleting an audio, etc), reminiscing (viewing a video, viewing a photo, etc), ecological momentary assessment (EMA) questions, and exit (logging out). Usage data across the course of the intervention were collected via secure email and statistically analyzed, and the findings have been published [31]. EMA is influenced by Kurt Lewin [32]. The use of "in-the-moment" approaches, along with rigorous measurement techniques in psychometric research, has been validated in recent research [33-36]. In our study, EMA involved the delivery of a small series of 5 questions directly to participants through the app; the feasibility of this approach in the context of dementia care is being appraised and will be reported in a subsequent paper. The InspireD system was designed with scalability in mind for future enhancements as it is envisaged that the final version will be a secure, cloud-based app accessible via a secure internet connection for authorized users. While the design incorporated the ability to store content locally on the device or to upload it to a cloud-based storage, a decision was made by the team that for the feasibility study, all content would be stored locally on the device.

\section{Methods}

\section{Design}

This paper reports on a feasibility study that incorporated a quasi-experimental design. An intervention of home-based personalized reminiscence, supported by a program of training and a novel iPad app, was examined for preliminary efficacy using 3 outcome measures pertaining to mutuality, emotional well-being, and quality of carer-patient relationships. The quasi-experimental design is appropriate and a common design for assessing the feasibility of novel technological interventions [37-39]. In line with quality standards, repeated measures testing was employed. Data were collected at baseline, midpoint, and intervention close. Table 1 outlines the intervention activities and data collection time-points.

The model of reminiscence that was utilized to underpin the training intervention was that of simple reminiscence [40], which encompasses mainly unstructured autobiographic storytelling and triggers that generate spontaneous reminiscence, often within a relational context, such as special days or events shared by friends and family. The goal of this approach is to enhance social contacts and short-term well-being while also supporting intergenerational bonding $[40,41]$. Our intervention was designed to cater for the needs, preferences, and interests of people living with dementia and their family carers. As a measure of quality, the reporting of this study adheres to the Transparent Reporting of Evaluations with Nonrandomized Designs (TREND) statement [42].

\section{Settings}

The setting was a large health and social care trust in a region of the United Kingdom. The trust catchment area is a mix of rural and urban communities serving a population of approximately 300,000 people, with an estimated 2717 of them living with dementia. Recruitment was facilitated by the trust's community mental health team for older people and through the trust's cognitive rehabilitation team as engagement with the latter was indicative of a diagnosis of early to moderate dementia.

\section{Participants}

A purposive sampling strategy was used to recruit 30 caregiving dyads ( 30 persons living with dementia and 30 carers). A sample size of 40-50 is recommended as sufficient for a feasibility study to estimate the total sample size across parameters to inform a future randomized controlled trial (RCT) [43]. Our participants were predominantly older people, and there was potential for significant dropout. With that in mind, we increased our sample size to 60 .

Inclusion and exclusion criteria were developed to minimize the potential for bias in the recruitment process. We included people who (1) had a diagnosis of early to moderate dementia, (2) were able to communicate and understand conversations, and (3) were aware of their dementia diagnosis; furthermore, we included family carers who were (1) aged $\geq 18$ years, (2) caring for a family member living with dementia meeting the above criteria (either cohabiting or non-cohabiting), and (3) aware of their relative's dementia diagnosis. Individuals with a major illness or disability that hindered their ability to engage in the study were excluded. Recruitment was commenced in April 2016 and continued until the sample size of 30 dyads ( $\mathrm{N}=60)$ was achieved in October 2016.

Ethical considerations principally pertained to voluntariness, supporting separate informed consent for the people living with dementia and their carers, handling and storage of data, and right to withdraw from the study. The study received ethical approval (REC Ref 16/NI/0002) in line with regional and National Health Service Trust research governance.

\section{Primary Outcome Measure}

The primary outcome measure was mutuality, defined as the positive quality of the relationship between the carer and the care recipient [26]. The Mutuality scale consists of 15 items. A sample item includes "How attached are you to him or her?" A 5-point scale is used, ranging from 0 (not at all) to 4 (a great deal). Higher scores indicate a higher level of mutuality, which may support relationships in difficult circumstances. The Mutuality scale has been tested for validity in previous studies of family caregiving and has demonstrated internal consistency $[44,45]$. The mean was calculated across the response scores for data analysis $[45,46]$.

\section{Secondary Outcome Measures}

The secondary outcome measures comprised QCPR [28] and WHO-5 [27,47]. The QCPR is a 14-item scale measuring relationship quality, including level of warmth and level of criticism. The scale has demonstrated good internal consistency and concurrent validity with other measures of relationship quality and carer stress [48]. Responses are rated using a 5-point Likert scale, scored from 1 (totally disagree) to 5 (totally agree). The 6 items measuring criticism and conflict are reverse scored in computation [28]. Total QCPR scores are utilized for data analysis. A total score $>42$ is indicative of a good relationship. 
The WHO-5 comprises 5 questions that tap into the subjective well-being of participants; it has been extensively tested for validity $[49,50]$ and reliability [51,52] scale items are scored from $0-5$ and then totaled, giving a potential raw score ranging from 0 to 25 . It is recommended that in studies assessing change over time, the WHO-5 raw scores are transformed to percentage scores for data analysis [53]. A total percentage score of $\leq 50$ is an indication of low mood, and a score of $\leq 28$ suggests likely depression, warranting further assessment.

\section{Intervention and Follow-Up}

Participating dyads were provided with a new touch screen tablet device that hosted the novel InspireD reminiscence app. A package of 5 reminiscence training sessions influenced by evidence-informed guidelines [40,41] was delivered by a reminiscence trainer employed by the Reminiscence Network Northern Ireland. Three information technology (IT) training sessions were then provided by an IT assistant to support the participants in uploading their personal memorabilia and to use the reminiscence app independently. The reminiscence and IT training packages were provided face-to-face, at the homes of participants living with dementia. The estimated cost of the intervention, which included the training package together with the cost of the InspireD system, was $£ 2570$ per dyad. After training was completed, participants were requested to engage in simple reminiscing through the app 3 days per week for the following 12 weeks. Compliance was supported by a user-friendly instruction booklet. A phone number for the IT trainer was provided, should any technological issues arise.
Participants were followed up for a period of 19 weeks from baseline $\left(\mathrm{T}_{0}\right)$, as outlined in Table 1. Midpoint measurement data $\left(\mathrm{T}_{1}\right)$ were collected on week 13 from baseline, which was 6 weeks into the independent use of the reminiscence technology. Endpoint measurement data were collected on week 19 at closure of the intervention $\left(\mathrm{T}_{2}\right)$. The data collection period was May 2016 to February 2017. Given the study design, there was no control group, and given the nature of the intervention, it was not possible to blind the participants or the trainers administering the intervention. All data were uploaded to IBM SPSS version 23 [54] using unique anonymized identification codes by the research assistant. The researcher responsible for analyzing the data and interpreting the results used that anonymized dataset.

\section{Statistical Analysis}

We used descriptive statistics to describe and synthesize the data pertaining to the characteristics of the participants. Missing data analysis, as recommended, was undertaken to discern possible patterns and challenges in the selected measurement tools [55]. We performed chi-square tests for analysis of nominal variables and independent $t$ tests to compare measurement scores in Mutuality, QCPR, and WHO-5 across the dyad relationship (people living with dementia and their carers) and across gender at baseline; chi-square tests were not performed when numbers in categories were less than 5. Paired $t$ tests were performed to investigate the differences in scores across 2 time-points. Furthermore, we performed within and between repeated measures analysis of variance (ANOVA) to investigate the impact of the intervention over time. 
Table 1. Intervention activities and data collection time-points.

\begin{tabular}{|c|c|c|}
\hline Timescale and activities & Data collection time-points & Repeated measures \\
\hline \multicolumn{3}{|l|}{ Preintervention } \\
\hline Baseline & Baseline $\left(\mathrm{T}_{0}\right)$ & $\begin{array}{ll}\text { - } & \text { Mutuality } \\
\text { - } & \mathrm{WHO}-5^{\mathrm{b}} \\
\text { - } & \mathrm{QCPR}^{\mathrm{c}}\end{array}$ \\
\hline \multicolumn{3}{|l|}{ 19-week intervention } \\
\hline \multicolumn{3}{|l|}{ Weeks 1-6 training } \\
\hline Reminiscence training package ( 5 sessions) & $\mathrm{N} / \mathrm{A}^{\mathrm{a}}$ & - $\quad$ N/A \\
\hline Information technology training package ( 3 sessions) & N/A & - $\quad$ N/A \\
\hline \multicolumn{3}{|l|}{ Week 7-19 reminiscence using iPad app } \\
\hline Home reminiscence begins in week 7 & N/A & - $\quad$ N/A \\
\hline Home reminiscence continues in week 13 & Midpoint $\left(\mathrm{T}_{1}\right)$ & $\begin{array}{ll}\text { - } & \text { Mutuality } \\
\text { - } & \text { WHO-5 } \\
\text { - } & \text { QCPR }\end{array}$ \\
\hline Home reminiscence ends in week 19 & Endpoint $\left(\mathrm{T}_{2}\right)$ & $\begin{array}{ll}\text { - } & \text { Mutuality } \\
\text { - } & \text { WHO-5 } \\
\text { - } & \text { QCPR }\end{array}$ \\
\hline
\end{tabular}

${ }^{\mathrm{a}} \mathrm{N} / \mathrm{A}$ : not applicable.

${ }^{b}$ WHO-5: World Health Organization-Five Well-Being Index.

${ }^{\mathrm{c}}$ QCPR: Quality of the Carer and Patient Relationship scale.

Correlational tests were performed to investigate relationships between continuous variables. On an intention-to-treat basis [55,56], missing data for Mutuality, QCPR, and WHO-5 were managed using the expectation-maximization imputation approach.

\section{Results}

\section{Baseline Assessment}

A baseline assessment of demographic details, Mutuality, QCPR, and WHO-5 was conducted prior to the program of reminiscence and IT training. We recruited 60 participants, in 30 dyads, in this study. Of them, a total of 58 participants (29 dyads) were retained in the study at completion. The participant characteristics and baseline measurement scores are presented in Table 2. Of all the participants with dementia, 67\% (20/30) were men. A chi-square test for independence (with Yates continuity correction) was performed, and it revealed that the

gender composition of the carer participants was different from that of participants living with dementia. Of all the carers, $80 \%$ $(24 / 30)$ were women $(P=.001)$. The age range of participants living with dementia was 61-94 years and that of the carers was 31-91 years. An independent $t$ test revealed that the age of the carers (mean 67 years, SD 14.8) was significantly lower than that of the participants living with dementia (mean 79 years, SD $8.9 ; P<.001)$. In 23 of the dyads, the carer was living in the same house as the participant living with dementia. The majority of the participants living with dementia had little or no IT experience, whereas the majority of carer participants had at least moderate IT experience.

There were no missing data at baseline. Mean mutuality score at baseline was 3.13 (SD 0.68), indicating a moderate level of closeness in the relationship. Visual inspection of the histogram and Q-Q plots indicated a reasonable but positively skewed distribution. 
Table 2. Baseline characteristics of participants.

\begin{tabular}{|c|c|c|c|c|}
\hline Characteristic & Number of participants $(\mathrm{N}=60)$ & People living with dementia $(\mathrm{n}=30)$ & Family carers $(\mathrm{n}=30)$ & $P$ value $^{\mathrm{a}}$ \\
\hline Age (years), mean (SD) & $73(13)$ & $79(8.9)$ & $67(14.8)$ & $<.001$ \\
\hline Age range (years) & $31-94$ & $61-94$ & $31-91$ & $\mathrm{~N} / \mathrm{A}^{\mathrm{b}}$ \\
\hline \multicolumn{5}{|l|}{ Gender, n (\%) } \\
\hline Male & $26(43)$ & $20(67)$ & $6(20)$ & N/A \\
\hline Female & $34(57)$ & $10(33)$ & $24(80)$ & .001 \\
\hline \multicolumn{5}{|l|}{ Marital status, $\mathrm{n}(\%)$} \\
\hline Married & $47(78)$ & $22(73)$ & $25(83)$ & N/A \\
\hline Widowed & $9(15)$ & $8(27)$ & $1(3)$ & N/A \\
\hline Separated or single & $4(6.7)$ & $0(0)$ & $4(13)$ & N/A \\
\hline \multicolumn{5}{|l|}{ IT $^{\mathrm{c}}$ experience, $\mathrm{n}(\%)$} \\
\hline Little or none & $35(58)$ & $24(80)$ & $11(37)$ & N/A \\
\hline Moderate & $21(35)$ & $5(17)$ & $16(53)$ & N/A \\
\hline A lot & $4(7)$ & $1(3)$ & $3(10)$ & N/A \\
\hline Home internet access, n (\%) & $52(87)$ & $25(83)$ & $27(90)$ & N/A \\
\hline \multicolumn{5}{|l|}{ Hobby choices, n (\%) } \\
\hline Social & $29(48)$ & $14(47)$ & $15(50)$ & N/A \\
\hline Physical fitness & $19(32)$ & $8(27)$ & $11(37)$ & N/A \\
\hline Creative & $7(12)$ & $4(13)$ & $3(10)$ & N/A \\
\hline No hobby & $5(8)$ & $4(13)$ & $1(3)$ & N/A \\
\hline \multicolumn{5}{|c|}{ Repeated measures, mean (SD) } \\
\hline Mutuality & $3.13(0.68)$ & $3.24(0.54)$ & $3.02(0.79)$ & .22 \\
\hline $\mathrm{QCPR}^{\mathrm{d}}$ & $57.4(7.9)$ & $58.1(7.1)$ & $56.7(8.6)$ & .52 \\
\hline WHO- $5^{\mathrm{e}}$ & $61.0(23.9)$ & $60.8(26.2)$ & $61.2(21.8)$ & .94 \\
\hline
\end{tabular}

${ }^{\mathrm{a}} P$ values $<.05$ indicate significance.

${ }^{\mathrm{b}} \mathrm{N} / \mathrm{A}$ : not applicable.

c IT: information technology.

${ }^{\mathrm{d}}$ QCPR: Quality of Carer-Patient Relationship scale.

${ }^{\mathrm{e}}$ WHO-5: World Health Organization-Five Well-Being Index.

There was no significant difference in baseline mutuality for participants living with dementia and carers $(P=.22)$. There was a statistically significant difference between mutuality for men (mean 2.9, SD 0.78) and women (mean 3.3, SD 0.56), with women having higher scores (95\% CI -0.756 to $-0.026 ; P=.04$ ). No relationship was discerned between age and baseline mutuality ( $\mathrm{r}=0.09)$.

Mean QCPR score was 57.4 (SD 0.9), indicating a good relationship. A Kolmogorov-Smirnov statistic of 0.057 was suggestive of a normal distribution, supported by visual inspection of histograms and Q-Q plots. There was no significant difference in baseline QCPR score between participants living with dementia and carers $(P=.52)$ or between men and women $(P=.91)$. There was a positive but weak correlation between age and QCPR ( $\mathrm{r}=-0.123)$, which failed to reach statistical significance $(P=.35)$.

Mean WHO-5 score was 61.0 (SD 23.9), indicating a moderate level of subjective well-being. The Shapiro-Wilks statistic (appropriate for a small sample) of 0.052 suggested normality, and this was supported by visual inspection of histograms and Q-Q plots. There was no significant difference in WHO-5 score between participants living with dementia and carers $(P=.94)$ or between men and women $(P=.40)$. No relationship was discerned between age and baseline WHO-5 $(\mathrm{r}=-0.04)$.

\section{Missing Data Analysis}

At $\mathrm{T}_{1}$ (midpoint), 5\% (3/60) participants had missing data: participant 50 had 2.9\% missing data; participant 11 had 5.9\% missing data, and participant 44 was unavailable for data collection due to a hospital admission. At $\mathrm{T}_{2}$ (endpoint), $6.6 \%$ (4/60) participants had missing data: participant 20 had 2.9\% missing data; participant 43 was unavailable due to a hospital admission, and one participant had died and her carer withdrew from the study. 


\section{Intention-to-Treat Analysis}

Paired-samples $t$ tests were conducted to compare baseline and endpoint measurement scores. There was a statistically significant increase in mutuality scores of participants living with dementia from baseline (mean 3.24, SD 0.545) to endpoint (mean 3.64, SD $0.274 ; 95 \%$ CI -0.56 to $-0.23 ; P<.001$, two-tailed). Furthermore, there was a statistically significant increase in QCPR scores of participants living with dementia from baseline (mean 58.07, SD 7.12) to endpoint (mean 63.2, SD $4.32 ; 95 \%$ CI -7.42 to $-2.84 ; P<.001$, two-tailed). Similarly, there was a statistically significant increase in WHO-5 scores of participants living with dementia from baseline (mean 60.8 , SD 26.2) to endpoint (mean 70.6 , SD $21.4 ; 95 \%$ CI -14.8 to $-4.84 ; P<.001$, two-tailed).

Regarding the carers, there was an increase in mutuality from baseline (mean 3.02, SD 0.79) to endpoint (mean 3.07, SD 0.60), but the increase was not statistically significant $(P=.52)$. Similarly, an increase in carer QCPR scores from baseline (mean 56.7, SD 8.66) to endpoint (mean 57.9, SD 8.26) was not statistically significant $(P=.28)$. There was a decrease in carer WHO-5 scores from baseline (mean 61.2, SD 21.8) to endpoint (mean 60.2, SD 23.4), but the change in the scores was not statistically significant $(P=.74)$.

Mixed between-within subjects ANOVA was performed to assess the impact of the reminiscence intervention over time and between participants living with dementia and the carers. For the participants living with dementia, mean mutuality increased from baseline to midpoint and then further increased at endpoint. For the carers, mean mutuality peaked at midpoint. A similar pattern for participants living with dementia and carers was observed in mean QCPR scores over time. Regarding WHO-5, the mean scores of participants living with dementia increased from baseline to midpoint, with a further increase at endpoint. For the carers, mean WHO-5 scores decreased from baseline to midpoint and then increased to the end point. Mean outcome measurement scores, SDs, time-related $P$ values, and pattern difference $P$ values are presented in Table 3 .
A statistically significant effect of the intervention on mutuality was demonstrated over time, Wilks lambda $=0.77, \mathrm{~F}(2,57)=8.17$, $P=.001$, partial eta squared $=0.22$. The pattern of mutuality scores for the participants living with dementia and the carers was significantly different, Wilks lambda $=0.87, \mathrm{~F}(2,57)=4.23, P=.02$, partial eta squared $=0.129$, with participants living with dementia exhibiting higher scores. A statistically significant effect of the intervention on QCPR was demonstrated over time, Wilks lambda $=0.777, \mathrm{~F}(2,57)=8.15, P=.001$, partial eta squared $=.223$. The pattern of QCPR scores for participants living with dementia and carers was statistically significant, Wilks lambda $=0.88$, $\mathrm{F}(2,57)=3.72, P=.03$, partial eta squared $=0.116$. The participants living with dementia exhibited higher scores. Overall, the intervention did not demonstrate a significant effect on WHO-5 scores over time, Wilks lambda $=0.90, \mathrm{~F}(2,57)=2.94, P=.06$, partial eta squared $=0.09$. However, a statistically significant difference was found in the pattern of scores between the participants living with dementia and the carers, Wilks lambda $=0.85, \mathrm{~F}(2,57)=4.90, P=.01$, partial eta squared $=0.147$. The participants living with dementia exhibited a pattern of higher scores.

\section{Estimation of Sample Size for a Future Randomized Controlled Trial}

A linear mixed model for a 2-way repeated measures ANOVA (fixed effects) was used to analyze the data. The between effect was dyad role, that is, participants living with dementia versus carers. The statistical power for the between effect in the model, based on the results from the mutuality measure, was 36 individuals per group (total=72). The power to detect the effects was set at 0.9 in all of the analyses.

For the within effect (repeated measures for both carers and those living with dementia), to detect the main effect of time (within subject effect), a sample of 16 respondents would be required in each group (total=32). For between-within subjects (interaction), to detect the interaction of condition (carer vs those living with dementia) and time, a sample of 39 individuals in each condition (total=78) would be required to detect an effect similar to that present in the previous study, with a statistical power of 0.9 . 
Table 3. Measures across time.

\begin{tabular}{|c|c|c|c|c|c|c|}
\hline Measure and participant & $\begin{array}{l}\text { Baseline }\left(\mathrm{T}_{0}\right) \text {, } \\
\text { mean }(\mathrm{SD})\end{array}$ & $\begin{array}{l}\text { Midpoint }\left(\mathrm{T}_{1}\right) \text {, } \\
\text { mean }(\mathrm{SD})\end{array}$ & $\begin{array}{l}\text { Endpoint, }\left(\mathrm{T}_{2}\right) \text {, } \\
\text { mean }(\mathrm{SD})\end{array}$ & $\mathrm{N}$ & Time $P$ value & Pattern $P$ value \\
\hline Mutuality & $3.1(0.687)$ & $3.4(0.55)$ & $3.4(0.543)$ & 60 & .001 & N/A \\
\hline PLWD $^{a}$ & $3.2(0.556)$ & $3.6(0.203)$ & $3.6(0.275)$ & 30 & $N / A^{b}$ & N/A \\
\hline Carer & $3.0(0.799)$ & $3.1(0.67)$ & $3.1(0.601)$ & 30 & N/A & .02 \\
\hline \multicolumn{7}{|l|}{$\mathrm{QCPR}^{\mathrm{c}}$} \\
\hline PLWD & $58.1(7.1)$ & $61.3(5.2)$ & $63.2(4.3)$ & 30 & N/A & N/A \\
\hline Carer & $56.8(8.7)$ & $58.6(7.4)$ & $58.9(8.3)$ & 30 & N/A & .03 \\
\hline Total & $57.4(7.9)$ & $59.9(6.5)$ & $60.6(7.0)$ & 60 & .001 & N/A \\
\hline \multicolumn{7}{|l|}{ WHO-5 ${ }^{d}$} \\
\hline PLWD & $60.8(26)$ & $69.9(18)$ & $70.7(21)$ & 30 & N/A & N/A \\
\hline Carer & $61.2(22)$ & $56.5(27)$ & $60.3(23)$ & 30 & N/A & .01 \\
\hline Total & $61.0(24)$ & $63.2(24)$ & $65.5(23)$ & 60 & .06 & N/A \\
\hline
\end{tabular}

${ }^{a}$ PLWD: person living with dementia.

${ }^{\mathrm{b}} \mathrm{N} / \mathrm{A}$ : not applicable.

${ }^{\mathrm{c}}$ QCPR: Quality of Carer-Patient Relationship scale.

${ }^{\mathrm{d}}$ WHO-5: World Health Organization-Five Well-Being Index.

\section{Discussion}

\section{Principal Findings}

In this quasi-experimental study, we sought to appraise outcomes from a feasibility study of individual-specific reminiscence facilitated by a program of training and an iPad app. A total of 58 participants (29 dyads) were retained in the study at completion, supporting the understanding that neither age nor a diagnosis of dementia are barriers to engagement in home-based research using technology. The main findings from the study are as follows: (1) statistically significant increases in mutuality, quality of carer and patient relationship, and emotional well-being of participants living with dementia from baseline to endpoint; (2) nonsignificant increases in mutuality and QCPR and a nonsignificant decrease in WHO-5 scores for the carer participants from baseline to endpoint; and (3) statistically significant differences in patterns of intervention effect across time, with the participants living with dementia exhibiting patterns of higher scores. It is difficult to determine the clinical significance of these changes as this was outside the remit of this feasibility study. However, a future RCT could include additional scales such as the mini-mental state examination [57] and Geriatric Depression Scale Short Form [58] in repeated measures testing.

\section{Comparison With Prior Work}

There is increasing use of mobile computer software and rising acceptability of health promotion apps internationally [29,59-62]. It was foreseen that the future would bring opportunities for reminiscing facilitated by touch screen interfaces [10]. Ours was a feasibility study with the objectives of testing a novel intervention of individual-specific reminiscence and investigating its impact. The total number of participants $(\mathrm{N}=60)$ recruited to this study is a significant increase from previous technology-enabled reminiscence studies [12]. The 3 outcome measures utilized in our study were mutuality [26], Quality of the Carer and Patient Relationship [28], and WHO-5 [47]. A strength of our study is that all 3 tools have previously undergone extensive testing for validity and reliability and were sufficiently sensitive to deliver statistically significant results. Our reminiscence intervention differed in a number of ways from the approaches taken in recent studies [12]: (1) our intervention was home based; (2) the participating dyads received a program of individual-specific training in reminiscing and IT, and (3) the reminiscing activity was supported by an iPad app hosted on tablet software with each of the participants having his or her own unique access log-in details. Our findings suggest that technology-based reminiscence may be able to support mutuality and quality of informal caregiving relationships, in contrast with negative trends observed in longitudinal studies among caregiving dyads $[22,44]$. We cannot make direct comparisons between our results and those of other technology-based reminiscence research due to the lack of appraisal of outcome in previous research. Our findings, however, add to the emerging evidence that technology-based reminiscence offers benefits in the context of family caregiving in dementia $[12,15,18]$.

It is acknowledged internationally that family carers are the most important practical, personal, and economic supports for people with dementia $[7,8]$ and that enduring caregiving roles in the context of dementia are associated with significant negative trends in mutuality and quality of life among family carers [22]. It would, therefore, not have been surprising if we had found significant negative trends in the outcome scores of carers over the course of our study. Our decision to deliver a home-based intervention was informed by research that suggested that research participation can pose a significant challenge to carers of people living with dementia [48]. Our 
research findings suggest that it is possible that the home-based nature of our intervention contributed to the statistically significant enhancements for participants with dementia, with no significant detriment to carers. To what extent carers would continue to support the intervention in a longer-duration study is worthy of consideration. It may be possible to develop the iPad app further to utilize a coaching companion to prompt, incentivize, and reward the carer. Taken together, our findings support the need for a robust RCT of home-based app-enabled personalized reminiscence. A stratified sampling strategy guided by mini-mental state examination scores, with matched controls, and a longer follow-up time of up to 2 years would address the unknown issue of how long the intervention effect might last.

\section{Limitations}

This was a feasibility study, and it was important to maximize the exposure to the novel technology-based personalized reminiscence intervention. There are acknowledged challenges in the recruitment of people living with dementia and their family carers, and use of a comparison group would have reduced exposure to the intervention. Quasi-experimental designs, such as the one we adopted, cannot establish cause-and-effect relationships with certainty, but they can establish strong links. We cannot rule out a Hawthorne effect given the trial design and the possibility that pre-existing factors have influenced the results. Conclusions, therefore, have to be interpreted with caution. An additional limitation is the underrepresentation of women among the participants living with dementia in our study, given that women have been constituted as a marginalized majority in UK prevalence of dementia [63].

\section{Conclusions}

Reminiscence has been promoted internationally as a means of enhancing standards of care and quality of life of people living with dementia and their family carers. Our study comprised a novel intervention of home-based reminiscence with repeated measures testing. The findings of this study indicate statistically significant enhancements in mutuality, quality of relationship, and subjective well-being for the participants living with dementia and nonsignificant enhancements in mutuality and quality of relationship for carers. These findings support an emerging body of evidence that purports that individual-specific psychosocial interventions have efficacy in the context of dementia. It is important to highlight that our study is not without limitations and that pre-existing factors may have influenced the results. Nonetheless, our intervention, comprising a program of training and use of a novel iPad app, may contribute to the ongoing development of home-based reminiscence in the context of dementia. Future research must be cognizant of the potential for women living with dementia to be underrepresented among participants and the importance of controlling pre-existing factors. A robust RCT of personalized reminiscence is worthy of consideration.

\section{Acknowledgments}

This work was funded by the Public Health Agency Research and Development Office and Atlantic Philanthropies (COM/5016/14). The authors would like to thank the Reminiscence Network Northern Ireland and people living with dementia and their carers for their input in the cocreation and design of the InspireD app and the Trust's Cognitive Rehabilitation Team for facilitating recruitment. Special thanks to Alex Turnbull from Kainos for invaluable assistance in early-stage technology selection decisions.

\section{Authors' Contributions}

EAL was responsible for study design, review board's approval, data collection, statistical analysis, interpretation of results, and manuscript preparation. AR was responsible for study design and management, review board's approval, recruitment, data collection, interpretation of results, and manuscript preparation. CM was responsible for recruitment, data collection, and manuscript preparation. RBB and MDM were responsible for EMA, design and analysis, interpretation of results, and manuscript preparation. KJC was responsible for study design, InspireD app development, and manuscript preparation. BB and FF were responsible for study design, interpretation of results, and manuscript preparation. AG was responsible for InspireD app development, data collection, and manuscript preparation.

\section{Conflicts of Interest}

None declared.

\section{References}

1. Prince M, Knapp M, Guerchet M, McCrone P, Prina M, Comas-Herrera A, et al. Alzheimer's Society. 2014 Nov. 2014 Dementia UK: Update 2nd edn URL: https://www.alzheimers.org.uk/download/downloads/id/2323/dementia uk update. pdf [WebCite Cache ID 6zHEhzxXZ]

2. Lawrence V, Fossey J, Ballard C, Moniz-Cook E, Murray J. Improving quality of life for people with dementia in care homes: making psychosocial interventions work. Br J Psychiatry 2012 Nov;201(5):344-351 [FREE Full text] [doi: 10.1192/bjp.bp.111.101402] [Medline: 23118034]

3. Gonzalez J, Mayordomo T, Torres M, Sales A, Meléndez JC. Reminiscence and dementia: a therapeutic intervention. Int Psychogeriatr 2015 Oct;27(10):1731-1737. [doi: 10.1017/S1041610215000344] [Medline: 25765779] 
4. Woods RT, Bruce E, Edwards RT, Elvish R, Hoare Z, Hounsome B, et al. REMCARE: reminiscence groups for people with dementia and their family caregivers - effectiveness and cost-effectiveness pragmatic multicentre randomised trial. Health Technol Assess 2012;16(48):v-xv, 1 [FREE Full text] [doi: 10.3310/hta16480] [Medline: 23211271]

5. Daly L, McCarron M, Higgins A, McCallion P. 'Sustaining Place' - a grounded theory of how informal carers of people with dementia manage alterations to relationships within their social worlds. J Clin Nurs 2013 Feb;22(3-4):501-512. [doi: 10.1111/jocn.12003] [Medline: 23170780]

6. Dassel KB, Carr DC, Vitaliano P. Does Caring for a Spouse With Dementia Accelerate Cognitive Decline? Findings From the Health and Retirement Study. Gerontologist 2017 Dec 01;57(2):319-328. [doi: 10.1093/geront/gnv148] [Medline: 26582383]

7. Hayes J, Zimmerman MK, Boylstein C. Responding to symptoms of Alzheimer's disease: husbands, wives, and the gendered dynamics of recognition and disclosure. Qual Health Res 2010 Aug;20(8):1101-1115. [doi: 10.1177/1049732310369559] [Medline: 20448273]

8. World Health Organization. 2017. Global action plan on the public health response to dementia 2017-2025 URL: http:/ lapps.who.int/iris/bitstream/handle/10665/259615/9789241513487-eng. pdf;jsessionid=DAF085809E514B4306EDD110F4A0A0F6?sequence=1[WebCite Cache ID 6zHEspu4I]

9. Gibson F. Reminiscence and life story work: A practice guide. London:: Jessica Kingsley Press; 2011.

10. Subramaniam P, Woods B. The impact of individual reminiscence therapy for people with dementia: systematic review. Expert Rev Neurother 2012 May;12(5):545-555. [doi: 10.1586/ern.12.35] [Medline: 22550983]

11. Karlsson E, Sävenstedt S, Axelsson K, Zingmark K. Stories about life narrated by people with Alzheimer's disease. J Adv Nurs 2014 Dec;70(12):2791-2799. [doi: 10.1111/jan.12429] [Medline: 24766325]

12. Lazar A, Thompson H, Demiris G. A systematic review of the use of technology for reminiscence therapy. Health Educ Behav 2014 Oct;41(1 Suppl):51S-61S [FREE Full text] [doi: 10.1177/1090198114537067] [Medline: 25274711]

13. Astell AJ, Ellis M, Bernardi L, Alm N, Dye R, Gowans G, et al. Using a touch screen computer to support relationships between people with dementia and caregivers. Interacting With Computers 2010;22(4):267-275.

14. Elfrink T, Juidema S, Kuinz M, Westerhof G. The effectiveness of creating an online life story book on persons with early dementia and their informal caregivers: a protocol of a randomized controlled trial. BMC Geriatr 2017;17 [FREE Full text] [Medline: 28431515]

15. Hamel AV, Sims TL, Klassen D, Havey T, Gaugler JE. Memory Matters: A Mixed-Methods Feasibility Study of a Mobile Aid to Stimulate Reminiscence in Individuals With Memory Loss. J Gerontol Nurs 2016 Jul 01;42(7):15-24 [FREE Full text] [doi: 10.3928/00989134-20160201-04] [Medline: 26870986]

16. Kerssens C, Kumar R, Adams AE, Knott CC, Matalenas L, Sanford JA, et al. Personalized technology to support older adults with and without cognitive impairment living at home. Am J Alzheimers Dis Other Demen 2015 Feb;30(1):85-97. [doi: 10.1177/1533317514568338] [Medline: 25614507]

17. Mulvenna M, Doyle L, Wright T, Zheng H, Topping P, Boyle K, et al. Evaluation of card-based versus device-based reminiscing use photographic images. J Cyber Ther Rehab 2011;4(1):57-66 [FREE Full text]

18. Haesner M, Steinert A, O'Sullivan JL, Weichenberger M. Evaluating an Online Cognitive Training Platform for Older Adults: User Experience and Implementation Requirements. J Gerontol Nurs 2015 Aug;41(8):22-31; quiz 32. [doi: 10.3928/00989134-20150710-44] [Medline: 26248140]

19. Bleakley CM, Charles D, Porter-Armstrong A, McNeill MDJ, McDonough SM, McCormack B. Gaming for health: a systematic review of the physical and cognitive effects of interactive computer games in older adults. J Appl Gerontol 2015 Apr;34(3):NP166-NP189. [doi: 10.1177/0733464812470747] [Medline: 24652863]

20. Delello JA, McWhorter RR. Reducing the Digital Divide: Connecting Older Adults to iPad Technology. J Appl Gerontol 2015 Jun 17. [doi: 10.1177/0733464815589985] [Medline: 26084479]

21. Regier NG, Gitlin LN. Dementia-related restlessness: relationship to characteristics of persons with dementia and family caregivers. Int J Geriatr Psychiatry 2018 Jan;33(1):185-192. [doi: 10.1002/gps.4705] [Medline: 28332736]

22. Farina N, Page TE, Daley S, Brown A, Bowling A, Basset T, et al. Factors associated with the quality of life of family carers of people with dementia: A systematic review. Alzheimers Dement 2017 May;13(5):572-581 [FREE Full text] [doi: 10.1016/j.jalz.2016.12.010] [Medline: 28167069]

23. McDonnell E, Ryan AA. The experience of sons caring for a parent with dementia. Dementia (London) 2014 Nov;13(6):788-802. [doi: 10.1177/1471301213485374] [Medline: 24339083]

24. Melunsky N, Crellin N, Dudzinski E, Orrell M, Wenborn J, Poland F, et al. The experience of family carers attending a joint reminiscence group with people with dementia: A thematic analysis. Dementia 2015;14(6):842-859.

25. McHugh JE, Wherton JP, Prendergast DK, Lawlor BA. Teleconferencing as a source of social support for older spousal caregivers: initial explorations and recommendations for future research. Am J Alzheimers Dis Other Demen 2012 Sep;27(6):381-387. [doi: 10.1177/1533317512453491] [Medline: 22871904]

26. Archbold PG, Stewart BJ, Greenlick MR, Harvath T. Mutuality and preparedness as predictors of caregiver role strain. Res Nurs Health 1990 Dec;13(6):375-384. [Medline: 2270302] 
27. Bech P, Olsen LR, Kjoller M, Rasmussen NK. Measuring well-being rather than the absence of distress symptoms: a comparison of the SF-36 Mental Health subscale and the WHO-Five Well-Being Scale. Int J Methods Psychiatr Res 2003;12(2):85-91. [Medline: 12830302 ]

28. Spruytte N, Van AC, Lammertyn F, Storms G. The quality of the caregiving relationship in informal care for older adults with dementia and chronic psychiatric patients. Psychol Psychother 2002 Sep;75(Pt 3):295-311. [Medline: 12396755]

29. Gibson A, McCauley C, Mulvenna M, Ryan A, Laird EA, Bunting B, et al. Assessing usability testing for people living with dementia. In: Rehab.: Rehab; 2016 Presented at: Proceedings of the 4th Workshop on ICTs for improving Patients Rehabilitation Research Techniques; 2016; Lisbon p. 25-31 URL: https://kevincurran.org/papers/ Accessing\%20Usability\%20Testing\%20for\%20People\%20with\%20Dementia\%20-\%20REHAB\%202016.pdf [doi: 10.1145/3051488.3051492]

30. Aydin M, Harmsen F, van SK, Stegwee R. On the Adaptation of an agile Information systems development method. J Database Management 2005;16(4):24-40.

31. Mulvenna M, Gibson A, McCauley CO, Ryan AA, Bond R, Laird EA, et al. Behavioural Usage Analysis of a Reminiscing App for People Living with Dementia and their Carers. In: Proceedings of the European Conference on Cognitive Ergonomics. 2017 Presented at: ECCE 2017; 2017; Umea, Sweden p. 35-38. [doi: 10.1145/3121283.3121289]

32. Lewin K. A dynamic theory of personality. New York: McGraw-Hill; 1935.

33. Place S, Blanch-Hartigan D, Rubin C, Gorrostieta C, Mead C, Kane J, et al. Behavioral Indicators on a Mobile Sensing Platform Predict Clinically Validated Psychiatric Symptoms of Mood and Anxiety Disorders. J Med Internet Res 2017 Mar 16;19(3):e75 [FREE Full text] [doi: 10.2196/jmir.6678] [Medline: 28302595]

34. Intille S, Rondoni J, Kukla C, Ancona I, Bao L. A context-aware experience sampling tool. In: Proceedings of CHI 2003. 2003 Presented at: CHI 2003; 2003; Lauderdale, Florida p. 972-973.

35. Mulder I, Ter HG, Kort J. SocioXensor: Measuring user behaviour and user experience in context with mobile devices,. In: Proceedings of Measuring Behavior. 2005 Presented at: The 5th International Conference on Methods and Techniques in Behavioral Research; 2005; Wageningen, The Netherlands p. 355-358.

36. Wiebe DJ, Nance ML, Houseknecht E, Grady MF, Otto N, Sandsmark DK, et al. Ecologic Momentary Assessment to Accomplish Real-Time Capture of Symptom Progression and the Physical and Cognitive Activities of Patients Daily Following Concussion. JAMA Pediatr 2016 Nov 01;170(11):1108-1110. [doi: 10.1001/jamapediatrics.2016.1979] [Medline: 27617669]

37. Kingston D, Janes-Kelley S, Tyrrell J, Clark L, Hamza D, Holmes P, et al. An integrated web-based mental health intervention of assessment-referral-care to reduce stress, anxiety, and depression in hospitalized pregnant women with medically high-risk pregnancies: a feasibility study protocol of hospital-based implementation. JMIR Res Protoc 2015 Jan 16;4(1):e9 [FREE Full text] [doi: 10.2196/resprot.4037] [Medline: 25595167]

38. Cook N, Winkler SL. Acceptance, Usability and Health Applications of Virtual Worlds by Older Adults: A Feasibility Study. JMIR Res Protoc 2016 Jun 02;5(2):e81 [FREE Full text] [doi: 10.2196/resprot.5423] [Medline: 27256457]

39. Toscos T, Daley C, Heral L, Doshi R, Chen Y, Eckert GJ, et al. Impact of electronic personal health record use on engagement and intermediate health outcomes among cardiac patients: a quasi-experimental study. J Am Med Inform Assoc 2016 Jan;23(1):119-128. [doi: 10.1093/jamia/ocv164] [Medline: 26912538]

40. Webster JD, Bohlmeijer ET, Westerhof GJ. Mapping the Future of Reminiscence: A Conceptual Guide for Research and Practice. Res Aging 2010 May 24;32(4):527-564. [doi: 10.1177/0164027510364122]

41. Kordelaar KV, Vlak A, Kuin Y, Westerhof G. Een handleiding en werkboek voor intergenerationele gesprekken [Time Traveling: A manual for intergenerational groups]. In: Nijmegen: Centre for Psychogerontology. Radboud Universiteit Nijmegen: Centrum voor Psychogerontologie; 2007:2007.

42. Des JDC, Lyles C, Crepaz N. Improving the reporting quality of nonrandomized evaluations of behavioral and public health interventions: the TREND statement. Am J Public Health 2004 Mar;94(3):361-366. [Medline: 14998794]

43. Sim J, Lewis M. The size of a pilot study for a clinical trial should be calculated in relation to considerations of precision and efficiency. J Clin Epidemiology 2012;65(3):301-308. [doi: 10.1016/j.jclinepi.2011.07.011] [Medline: 22169081]

44. Lyons KS, Sayer AG, Archbold PG, Hornbrook MC, Stewart BJ. The enduring and contextual effects of physical health and depression on care-dyad mutuality. Res Nurs Health 2007 Feb;30(1):84-98. [doi: 10.1002/nur.20165] [Medline: 17243110]

45. Tetz KB, Archbold PG, Stewart BJ, Messecar D, Hornbrook MC, Lucas SA. How frail elders evaluate their caregiver's role enactment: a scale to measure affection, skill, and attentiveness. J Fam Nurs 2006 Aug;12(3):251-275. [doi: 10.1177/1074840706291554] [Medline: 16837694]

46. Schumacher KL, Stewart BJ, Archbold PG. Mutuality and preparedness moderate the effects of caregiving demand on cancer family caregiver outcomes. Nurs Res 2007;56(6):425-433. [doi: 10.1097/01.NNR.0000299852.75300.03] [Medline: 18004189]

47. World Health Organization. 1998. Wellbeing Measures in Primary Health Care/The Depcare Project URL: http://www. euro.who.int/ data/assets/pdf file/0016/130750/E60246.pdf[WebCite Cache ID 6yihIEpLT] 
48. Woods RT, Orrell M, Bruce E, Edwards RT, Hoare Z, Hounsome B, et al. REMCARE: Pragmatic Multi-Centre Randomised Trial of Reminiscence Groups for People with Dementia and their Family Carers: Effectiveness and Economic Analysis. PLoS One 2016;11(4):e0152843 [FREE Full text] [doi: 10.1371/journal.pone.0152843] [Medline: 27093052]

49. Henkel V, Mergl R, Kohnen R, Allgaier A, Möller H, Hegerl U. Use of brief depression screening tools in primary care: consideration of heterogeneity in performance in different patient groups. Gen Hosp Psychiatry 2004;26(3):190-198. [doi: 10.1016/j.genhosppsych.2004.02.003] [Medline: 15121347]

50. Liwowsky I, Kramer D, Mergl R, Bramesfeld A, Allgaier A, Pöppel E, et al. Screening for depression in the older long-term unemployed. Soc Psychiatry Psychiatr Epidemiol 2009 Aug;44(8):622-627. [doi: 10.1007/s00127-008-0478-y] [Medline: $\underline{19048174]}$

51. de WM, Pouwer F, Gemke RJBJ, Delemarre-van DWHA, Snoek FJ. Validation of the WHO-5 Well-Being Index in adolescents with type 1 diabetes. Diabetes Care 2007 Aug;30(8):2003-2006. [doi: 10.2337/dc07-0447] [Medline: 17475940]

52. Löwe B, Spitzer RL, Gräfe K, Kroenke K, Quenter A, Zipfel S, et al. Comparative validity of three screening questionnaires for DSM-IV depressive disorders and physicians' diagnoses. J Affect Disord 2004 Feb;78(2):131-140. [Medline: 14706723]

53. Topp CW, Østergaard SD, Søndergaard S, Bech P. The WHO-5 Well-Being Index: a systematic review of the literature. Psychother Psychosom 2015;84(3):167-176 [FREE Full text] [doi: 10.1159/000376585] [Medline: 25831962]

54. IBM. IBM SPSS Statistics for Windows, Version 23.0. Armonk, New York: IBM Corporation; 2016.

55. Bannon W. Missing data within a quantitative research study: How to assess it, treat it, and why you should care. J Am Assoc Nurse Pract 2015 Apr;27(4):230-232. [doi: 10.1002/2327-6924.12208] [Medline: 25676704]

56. Pallant J. SPSS Survival Manual. New York: McGraw Hill; 2010.

57. Folstein MF, Folstein SE, McHugh PR. "Mini-mental state". A practical method for grading the cognitive state of patients for the clinician. J Psychiatr Res 1975 Nov;12(3):189-198. [Medline: 1202204]

58. Sheikh J, Yesavage J. Geriatric Depression Scale (GDS): Recent evidence and development of a shorter version. Clin Gerontol 1986;5(1/2):165-173 Published online: 25 Oct 2008.

59. Kerkhof YJF, Graff MJL, Bergsma A, de VHHM, Dröes R. Better self-management and meaningful activities thanks to tablets? Development of a person-centered program to support people with mild dementia and their carers through use of hand-held touch screen devices. Int Psychogeriatr 2016 Dec;28(11):1917-1929. [doi: 10.1017/S1041610216001071] [Medline: 27425002]

60. Krebs P, Duncan DT. Health App Use Among US Mobile Phone Owners: A National Survey. JMIR Mhealth Uhealth 2015;3(4):e101 [FREE Full text] [doi: 10.2196/mhealth.4924] [Medline: 26537656]

61. Becker S, Miron-Shatz T, Schumacher N, Krocza J, Diamantidis C, Albrecht U. mHealth 2.0: Experiences, Possibilities, and Perspectives. JMIR Mhealth Uhealth 2014;2(2):e24 [FREE Full text] [doi: 10.2196/mhealth.3328] [Medline: 25099752]

62. Hale K, Capra S, Bauer J. A Framework to Assist Health Professionals in Recommending High-Quality Apps for Supporting Chronic Disease Self-Management: Illustrative Assessment of Type 2 Diabetes Apps. JMIR Mhealth Uhealth 2015;3(3):e87 [FREE Full text] [doi: 10.2196/mhealth.4532] [Medline: 26369346]

63. Alzheimer's Research UK. Women and dementia: A marginalised majority. Cambridge: Alzheimer's Research UK; 2015. URL: https://www.alzheimersresearchuk.org/wp-content/uploads/2015/03/Women-and-Dementia-A-Marginalised-Majority1. pdf [accessed 2018-08-08] [WebCite Cache ID 71rtBEgrl]

\author{
Abbreviations \\ ANOVA: analysis of variance \\ EMA: ecological momentary assessment \\ ICT: information and communication technology \\ InspireD: Individual-Specific Reminiscence for People living with Dementia \\ IT: information technology \\ QCPR: Quality of Carer-Patient Relationship scale \\ RCT: randomized controlled trial \\ UI: user interface \\ WHO: World Health Organization \\ WHO-5: World Health Organization-Five Well-Being Index
}


Edited by $G$ Eysenbach; submitted 19.12.17; peer-reviewed by ME Kamasak, $Q$ Yuan, $R$ El Shafie, K Hale; comments to author 18.03.18; revised version received 10.05.18; accepted 18.06.18; published 11.09.18

Please cite as:

Laird EA, Ryan A, McCauley C, Bond RB, Mulvenna MD, Curran KJ, Bunting B, Ferry F, Gibson A

Using Mobile Technology to Provide Personalized Reminiscence for People Living With Dementia and Their Carers: Appraisal of Outcomes From a Quasi-Experimental Study

JMIR Ment Health 2018;5(3):e57

URL: http://mental.jmir.org/2018/3/e57/

doi: $\underline{10.2196 / \text { mental. } 9684}$

PMID: $\underline{30206053}$

CElizabeth A Laird, Assumpta Ryan, Claire McCauley, Raymond B Bond, Maurice D Mulvenna, Kevin J Curran, Brendan Bunting, Finola Ferry, Aideen Gibson. Originally published in JMIR Mental Health (http://mental.jmir.org), 11.09.2018. This is an open-access article distributed under the terms of the Creative Commons Attribution License (https://creativecommons.org/licenses/by/4.0/), which permits unrestricted use, distribution, and reproduction in any medium, provided the original work, first published in JMIR Mental Health, is properly cited. The complete bibliographic information, a link to the original publication on http://mental.jmir.org/, as well as this copyright and license information must be included. 